\title{
Introduction: High Resolution Cryo-SEM in the Biological Sciences
}

What is cryogenic high resolution (in-lens) scanning electron microscopy (HRSEM), and do I need it? Structural cell biologists and bioorganic chemists will find this newly developed imaging mode to be an accurate and useful research tool for molecular level investigations in the hydrated state.

The ultimate use of this type of FESEM, versus a below-lens FESEM fitted with a cryo-stage, is for topographic resolution of biomolecular fine structure and supramolecular complexes. The in-lens microscope requires a smaller sample size and a separate dedicated preparation system. In-lens HRSEMs collect specimen-specific secondary electrons that ensure the highest spatial resolution of the frozen biological surface. While the sample areas to be examined may seem vast in microscopy terms (spanning 1-2 $\mathrm{mm}^{2}$ ), the structures of interest are only visible at magnifications above 50,000×. Below-lens stages can have cryo-preparation systems built onto the airlock of the chamber and can accommodate larger specimens, but at a more modest level of resolution.

Cryo in-lens HRSEMs use a dedicated cryo-stage identical to those used for cryo-TEM and can also be used for cryo-STEM in tandem with HRSEM. What is critical to both types of cryo-SEM imaging (in-lens and below-lens) is that the images of the sample must accurately represent the structure in its native aqueous state. To this end, rapid freezing methods, particularly high pressure freezing (HPF), are essential procedures for specimen immobilization and minimization of dimensional changes that occur in conventional preparations due to the removal of water. Dedicated systems facilitate cryo-specimen processing such as cryo-fracturing, cryo-sectioning, etching, and metal coating. These procedures work best with small pieces of biological tissue or biomaterial systems such as hydrogels, the same small specimen sizes that are suited to the in-lens cryo-specimen stage. Metal coating can enrich SE-I contrasts or BSE-I contrasts, particularly for instruments with high-angle BSE detectors.

The authors of three cryo-HRSEM papers in this issue present their methods and strategies for "quality" imaging of hydrated biological systems. These papers are published in conjunction with a symposium entitled "High Resolution Cryo-SEM in the Biological Sciences" at Microscopy and Microanalysis 2003 in San Antonio, Texas, August 4-7, 2003.

Robert P. Apkarian 